\title{
Significado del puerperio en pandemia por COVID-19: nueva dimensión del cuidado
}

\section{Meaning of the puerperium in a pandemic due to COVID-19: a new dimension of care}

\author{
Patricia Lapeira-Panneflex (iD) ${ }^{1}$, Edna Osorio-Duran (iD) ${ }^{2}$, Rosemary Castañeda-Mercado (iD) 3 \\ 1. Universidad del Magdalena. Santa Marta, Colombia. Correo: plapeira@unimagdalena.edu.co - https://orcid.org/0000-0002-1088-13452 \\ 2. Universidad del Magdalena. Santa Marta, Colombia. Correo: eosoriod@unimagdalena.edu.co - https://orcid.org/0000-0003-4566-3118 \\ 3. Universidad del Magdalena. Santa Marta, Colombia. Correo: rcastañeda@unimagdalena.edu.co - https://orcid.org/0000-0002-0513-2235
}

Tipología: Artículo de investigación científica y tecnológica

Para citar este artículo: Lapeira-Panneflex P, Osorio-Durán E, Castañeda-Mercado R. Significado del puerperio en pandemia por COVID-19: nueva dimensión del cuidado. Duazary. 2021 septiembre; 18(3 número especial): 91-99. Doi: https://doi.org/10.21676/2389783X.4273

Palabras clave: puerperio; autocuidado; COVID-19.

\section{RESUMEN}

La emergencia sanitaria por la COVID-19 ha cambiado la dinámica del cuidado. El puerperio es una etapa de muchos cambios y supone vulnerabilidad en este grupo de mujeres, que por causas de las medidas de aislamiento no poseen apoyo social. El propósito de este estudio fue describir el significado del cuidado en el puerperio para la mujer en situación de pandemia. Se empleó la etnoenfermería (Leininger) con la entrevista no estructurada como método de recolección de los datos. Participaron en el estudio 8 puérperas como informantes claves, 2 madres y 2 suegras como informantes generales. Para el análisis de la información se utilizó el método de Spradley", el cual incluye los dominios, taxonomías, análisis componencial y temas. Los dominios resultantes: cuidarse la mujer durante el puerperio hoy es la manera de asegurar su salud en el futuro, cuidar a su hijo es la manera de evitar que se enferme. Se concluye que las mujeres puérperas adquieren con el contacto en su grupo social diferentes conceptos del cuidado, que por motivos de las medidas de aislamiento buscan fuentes de información como el internet, incorporándolas a su cotidianidad propagando creencias, costumbres y que se mantienen con el transcurso del tiempo.

\section{ABSTRACT}

Keywords: Puerperium; Self-care; COVID-19. he health emergency due to the coronavirus covid 19 has changed the dynamics of care. The puerperium is a stage of many changes and implies vulnerability in this group of women, who due to isolation measures do not have social support. The purpose of this study was to describe the meaning of postpartum care for women in a pandemic situation. Ethno-nursing (Leininger) was used with the unstructured interview as the data collection method. Eight puerperal women participated in the study as key informants, 2 mothers and 2 mothers-in-law as general informants. For the analysis of the information, the Spradley method was used", which includes the domains, taxonomies, component analysis and themes. The resulting domains: taking care of the woman during the puerperium today is the way to ensure her health in the future, taking care of her child is the way to prevent her from getting sick. It is concluded that puerperal women acquire different concepts of care with contact in their social group, which for reasons of isolation measures seek sources of information such as the internet, incorporating them into their daily lives spreading beliefs, customs and that are maintained over time. weather. 


\section{INTRODUCCIÓN}

Durante la pandemia por COVID-19 las mujeres en posparto y sus neonatos han sido considerados uno de los grupos prioritarios de estudio en relación con el coronavirus ${ }^{1}$. El puerperio es una etapa que amerita un cuidado centrado en las necesidades de cada paciente, durante este período las puérperas ejecutan prácticas de cuidado, buscando garantizar el mantenimiento de su salud y la del recién nacido ${ }^{2}$ Con frecuencia se considera como un estado normal que no requiere de mucha atención. Sin embargo, no podemos olvidar que muchos de los cambios que presenta la madre, aunque son normales, no dejan de ser estados fisiológicos, potencialmente peligrosos $^{3}$ aunado a esto las puérperas tienen un riesgo de enfermedad tromboembólica venosa superior al resto de las mujeres por lo que la infección con el nuevo coronavirus SARSCoV-2 (COVID-19), debe considerarse como un factor de riesgo más para la enfermedad tromboembólica venosa en este grupo. En cuanto a la trasmisión vertical del virus, aún no se evidencia información científica suficiente que sustente este hecho ${ }^{4}$, dado que existe escasa literatura científica sobre el coronavirus en la población de mujeres en posparto y sus recién nacidos.

La mujer en el puerperio tiende a mantener sus redes sociales de apoyo, pero también la amplía con otras mujeres que hayan tenido la experiencia de la maternidad, como la madre, suegra tía o abuela por tener mayor experiencia del cuidado en el puerperio, quienes tradicionalmente se trasladan a la casa de la nueva madre, para ayudarle en los cuidados de ella y su recién nacido, lo cual fortalece sus conocimientos y prácticas para cuidarse. El cuidado que realiza la puérpera se encuentra inmerso en un legado cultural que es trasmitido de generación en generación ${ }^{5}$ en donde existe un respeto por la jerarquía femenina y se tiene en cuenta estos cuidados para la conservación de la salud de la mujer en esta etapa. Las puérperas adquieren con el contacto de su grupo social diferentes conceptos y formas de cuidado, los cuales incorpora a su cotidianidad propagando creencias y costumbres que se mantienen con el transcurso del tiempo.
La orientación de este cuidado ha cambiado, en especial en estos momentos en que por motivos de la emergencia sanitaria generada por el covid-19 para evitar la rápida propagación del virus, se está produciendo un egreso temprano de las puérperas a sus hogares dentro de las ocho y doce horas después del parto y la atención posparto se realiza de forma virtual ${ }^{6}$. El acompañamiento de las mujeres de experiencia en el cuidado dentro de la familia, en el hogar, como lo es la abuela, suegra, tía o madre de la puérpera, en muchos casos no es posible durante la pandemia por diversos motivos, ya sea por pertenecer a la tercera edad, presentar alguna comorbilidad o por restricciones de movilidad debido a las medidas de distanciamiento social en constante cambio, para lograr controlar los casos en este grupo etario.

En este período y bajo las actuales circunstancias al encontrarse la puérpera en su hogar, sin un asesoramiento efectivo del equipo de salud y el distanciamiento de sus redes de apoyo social familiar, acude a buscar información en las redes sociales disponible, lugar que le permite también compartir la experiencia con otras madres aumentando su empoderamiento en el nuevo rol de madre.

Por lo anteriormente descrito esta investigación tiene como referente teórico la teoría de los cuidados culturales de Madeleine Leinninger la cual considera a los seres humanos como inseparables de su trasfondo cultural y su estructura social, afirma:" describir como las culturas tienen diferentes modelos de cuidado y maneras diferentes para sentirse bien y enfermarse es un asunto vital para la enfermería?.

En situación de aislamiento social la mujer puérpera realiza su propio cuidado, en su hogar el cual no es conocido por parte de enfermería. Siendo el cuidado el objeto focal de la práctica de enfermería. El objetivo por investigar fue describir el significado del cuidado en el puerperio para la mujer en situación de pandemia. 


\section{MATERIALES Y MÉTODOS}

\section{Diseño}

La investigación cualitativa fue utilizada en este estudio por ser un diseño emergente que admite una visión holística del fenómeno, y que requiere del investigador una actitud flexible ${ }^{8}$ con el diseño etnográfico presentado por Spradley ${ }^{9}$ debido a que se orienta a describir significativamente actitudes de la comunidad dentro de un fenómeno específico, en este caso el puerperio y como lo define Leininger ${ }^{10}$ es una etnografía de pequeña escala, porque se busca definir un fenómeno en un grupo clave y se centra en un aspecto en particular que son los significados.

\section{Participantes}

Los informantes claves de esta investigación, considerados aquellos que "generalmente tienen más conocimiento de su cultura y los más interesados en el dominio de investigación" ${ }^{11}$ fueron 8 puérperas.

Los informantes generales: son los que "usualmente tienen solo conocimiento general sobre el dominio de investigación; sin embargo, tienen alguna comprensión sobre el tema de la investigación y pueden llegar a compartir visiones relevantes y puntos de vista culturales"11. Para este estudio los informantes generales fueron 4 conformados por 2 madres y 2 suegras. Para proteger la identidad de nuestras informantes, sus nombres fueron remplazados por nombre de flores.

Los criterios de inclusión fueron ser puérpera entre 0 y 42 días posparto y que quisieran participar voluntariamente luego de dar a conocer los objetivos de esta investigación.

El criterio utilizado para determinar el número de entrevistas a realizar fue el de saturación de datos. El cual se obtiene cuando se recolecta la información hasta el punto en que ya no se consigue información nueva y la que se tenía comienza a repetirse ${ }^{12}$.

\section{Instrumentos}

La información se obtuvo a través de la entrevista no estructurada, Entre los tipos de preguntas se utilizaron: Preguntas descriptivas, por ejemplo: ¿Cuál es su rutina en un día común y corriente de su vida ahora? Preguntas estructurales, por ejemplo: ¿De quién recibe la información sobre los cuidados para usted en esta etapa y para su bebé? Preguntas de contraste, por ejemplo: ¿Qué aportes en cuanto a la información recibida para su cuidado en el posparto y el de su bebé, de diversas fuentes (personas, redes sociales, etc.) considera usted que le han sido útiles? Estos tres tipos de preguntas permiten una aproximación a diferentes momentos en el proceso de acercamiento sobre un tema o problema, el conocimiento de la nueva información, la comprobación y la relación en los que se toman decisiones, en otras palabras, se considera que con las preguntas descriptivas el investigador intenta acercarse al contexto en que el informante desarrolla sus actividades rutinarias; las preguntas estructurales se formulan para comprobar las explicaciones extraídas de los informantes, estas permiten al entrevistador comprobar la interpretación que ha hecho de los significados que emplea su informante; y las preguntas de contraste se emplean para extraer diferencias entre los términos utilizados por un informante, los cuales permiten descubrir las relaciones tácitas entre los mismos $^{13}$.

Dada la actual emergencia sanitaria por covid-19, se concertó una cita por WhatsApp, luego se realizó una llamada telefónica en donde se explicó el objetivo del estudio y posteriormente fue enviando por correo electrónico el consentimiento informado, finalmente se efectuó la entrevista a través de video llamada con el fin de observar su expresión y lenguaje corporal de las participantes, las cuales fueron grabadas en audio y luego transcritas. El análisis de la información suministrada por las puérperas fue realizado en forma simultánea, las entrevistas fueron verificadas en su totalidad por las investigadoras.

\section{Procedimiento}

El análisis de la información se realizó en forma simultánea con la recolección de la información y se 
manejó la propuesta metodológica de análisis etnográfico que se utilizó en la presente investigación fue desarrollada por James Spradley ${ }^{14}$ el cual incluye: Dominios, taxonomías, análisis componencial y temas. Según este autor descubrir dominios implica buscar similitudes existentes entre los términos. El análisis de dominio implica hacer uso de las relaciones semánticas. Se identificaron los dominios y luego se pasó a la construcción de taxonomías, estas últimas para la actual investigación utilizando el método de Spradley, son una aproximación al cuidado de las puérperas según su conocimiento cultural en situación de pandemia, y posteriormente emergen los temas. Cada uno de los dominios incluyó los tres elementos básicos: el término cubierto o dominio cultural, los términos incluidos y la relación semántica, que es la encargada de establecer una conexión entre el término incluido y el nombre del dominio. Finalmente, se descubren los temas culturales, a partir de ellos se facilitó establecer, por parte de las investigadoras, las conclusiones y recomendaciones.

Tabla 1. Tipo de relaciones semánticas según James Spradley.

\begin{tabular}{|l|l|}
\hline Relación semántica & Expresión \\
\hline Relación causa efecto & X es el resultado de $\mathrm{Y}$ \\
\hline Relación medio fin & $\begin{array}{l}\mathrm{X} \text { es una manera de tener } \\
\mathrm{Y}\end{array}$ \\
\hline $\begin{array}{l}\text { Relación de inclusión } \\
\text { estricta }\end{array}$ & $\mathrm{X}$ es una clase de $\mathrm{Y}$ \\
\hline $\begin{array}{l}\text { Relación de justificación } \\
\text { o de razón }\end{array}$ & X es una razón de $\mathrm{Y}$ \\
\hline Relación de atribución & $\begin{array}{l}\mathrm{X} \text { es una característica de } \\
\mathrm{Y}\end{array}$ \\
\hline
\end{tabular}

Fuente: Spradley J. La entrevista etnográfica ${ }^{14}$.

En cuanto al rigor metodológico para garantizar la calidad científica en esta investigación se tuvieron en cuenta los criterios de Castillo y Vásquez ${ }^{15}$ la credibilidad, la auditabilidad y la transferibilidad. La credibilidad se aseguró a través de la confrontación de las interpretaciones con las participantes del estudio y el acompañamiento permanente de expertos en la temática que contribuyeron a cambiar la perspectiva inicial de las investigadoras. La auditabilidad o confirmabilidad se alcanzó por medio del seguimiento de la ruta de la investigación, dado que se grabaron las entrevistas de las participantes, se realizó la transcripción textual de las mismas de la confrontación de las interpretaciones con las participantes del estudio. La transferibilidad o aplicabilidad se posibilita en la medida que se describa de manera detallada el diseño y el marco de análisis para que distintos investigadores repliquen el estudio con nuevas poblaciones y en otros contextos culturales.

\section{Declaración sobre los aspectos éticos}

En este estudio se tuvo en cuenta los principios éticos consagrados en la resolución 008430 del 4 de octubre de 1993 en la cual se establecen las normas científicas, técnicas y administrativas para la investigación en salud ${ }^{16}$, para lo cual se elaboró consentimiento informado, entregado por correo electrónico, previa explicación de los objetivos y beneficios que se esperan lograr de la investigación, a través de llamada telefónica, con anterior concertación de la cita por WhatsApp, garantizando además la confidencialidad de la información obtenida que acepten de manera voluntaria participar en el estudio y cumplan con los criterios de inclusión.

\section{RESULTADOS}

\section{Características sociodemográficas de las participantes}

Las informantes claves fueron 8 puérperas entre los 20 y 26 años de edad, 6 de ellas ya habían tenido un hijo anteriormente y 2 tenían la experiencia del puerperio por primera vez. En cuanto a la escolaridad 2 profesionales, 4 estudios técnicos y 2 con bachillerato incompleto. Estado civil: 6 en unión libre y 2 casadas. Ocupación: todas amas de casa y de estrato socioeconómico 3. Todas afiliadas al régimen contributivo en salud.

Las informantes generales fueron 4: 2 madres y 2 suegras, en edades entre los 42 y 67 años. Estado civil: casadas:2, viuda:1 y 1 en unión libre. Escolaridad: 2 primaria completa y 2 secundaria incompleta. Todas amas de casa, de estrato socioeconómico 3 y afiliadas al régimen contributivo de salud. 


\section{Dominio Cultural 1: Forma de cuidarse la mujer durante el puerperio hoy es la manera de asegurar su salud en el futuro}

Las puérperas entrevistadas consideran que cuidarse hoy es la manera de asegurar su salud en el futuro, durante esta etapa, según ellas afirman que es preciso no alzar peso, no salir a la calle a serenarse y tomar bebidas calientes para garantizar el no enfermarse más adelante, ya que el puerperio es un periodo caliente y se debe evitar que entre frío al cuerpo.

"Yo no me sereno, ni salgo a la calle después que se oculta el sol, y ahora mucho menos con esto de la pandemia, tomo agua de panela caliente y avena para que me baje bastante leche" Azucena

"A mi particularmente me gusta el pescado, pero no lo como durante la dieta, porque es un alimento frio porque viene del mar para que no me entre frío al cuerpo y eso no sale ahora, pero con los años vienen los problemas de salud el dolor de cabeza, en la cintura, las piernas...en fin" Margarita

La fuente de información que utilizan las puérperas para los cuidados que se propician ellas y los de su recién nacidos son las madres, abuelas suegras y las redes sociales dado que la familia en ocasiones no se puede trasladar a su hogar por la emergencia sanitaria como es la costumbre en estos casos. De otro lado algunas manifestaron utilizar las redes sociales para la búsqueda de información referente al cuidado de los recién nacidos

"Bueno, mi mamá no pudo venir a cuidarme a mi casa porque ella tiene 69 años, y las personas de más edad deben cuidarse más en la pandemia, entonces a veces viene mi hermana mayor, pero no es lo mismo. Aunque hablamos todos los días por teléfono" Girasol

"Mi suegra me llama, yo le pregunto cualquier cosa en la que tenga dudas con respecto al cuidado del niño y en ocasiones consulto en internet, algunas redes sociales y como estoy sola pues me toca hacer oficio, aunque yo sé que no debo porque estoy en posparto" Rosa

\section{Dominio cultural 2: Cuidar a su hijo es la manera de evitar que se enferme}

El cuidado del recién nacido es asunto importante para la madre por eso se aseguran de abrigarlo bien, no sacarlo a la calle, no aceptar visitas puesto que pueden llevar enfermedades, baño diario y una buena alimentación con leche materna son los principales cuidados.

"Yo sé que debo cuidarlo para que no se enferme lo abrigo bien sobretodo en su mollerita porque ir a una clínica en estos momentos es peligroso, el coronavirus está más en esos lugares" Rosa

"Uno del cuidado que yo tengo con la bebé es darle leche materna los protege de enfermedades" Margarita

\section{Dominio cultural 3: Demasiada información es la razón del miedo}

Las entrevistadas manifiestan que la emergencia sanitaria por covid-19 ha traído mucha información en redes sociales, noticieros y demás medios masivos de comunicación sobre el tema, por lo cual ellas sienten miedo de enfermarse y trasmitir a sus recién nacidos, la enfermedad.

"La verdad es que yo tengo un poco de miedo de enfermarme en estos momentos porque si yo me enfermo ¿quién cuida a mi hijo?" Rosa

"Es que dicen tantas cosas en los noticieros, redes sociales, que uno no sabe que es cierto y qué no respecto al virus" Azucena

Algunas puérperas manifiestan sentirse solas y cansadas, ya que por la situación de emergencia sanitaria sus familiares no han podido estar con ellas, razón por la cual también sienten miedo de no saber cuidar bien al bebé o enfermarse ellas.

"Es duro estar sola aquí y con el cuidado del bebé, se cansa uno más, aunque uno entiende que es por el bien de todos" Girasol. 


\section{DISCUSIÓN}

Leininger define el cuidado como las experiencias o ideas de asistencia, apoyo y facilitación hacia otros con necesidades evidentes o anticipadas para mejorar las condiciones humanas o el estilo de vidas $^{17}$. El cuidado de las puérperas y sus recién nacidos han sido considerados como prioritarios en la actual emergencia sanitaria ${ }^{18}$

Las madres entrevistadas manifestaron que los cuidados realizados durante el puerperio y lo suministrado a sus recién nacidos provienen del conocimiento cultural adquiridos en el contacto con su grupo familiar principalmente con las representantes del género femenino: madre, suegra y hermana mayor, hallazgo que coincide con otras investigaciones realizada en este grupo etario $^{19,20}$

En las actuales circunstancias de confinamiento por la emergencia sanitaria propiciada por la covid-19 las puérperas entrevistadas indagan en las redes sociales, convirtiéndose estas en una fuente de información, según investigaciones realizadas el acceso a información y experiencias compartidas pueden ser particularmente enriquecedoras durante los períodos posteriores al nacimiento, especialmente para las madres que descubren que su red física inmediata no satisface sus necesidades de información o apoyo en el hogar ${ }^{6,21}$, aumentando el sentido real de empoderamiento y ayudando a mejorar el desafío de convertirse en madre ${ }^{22}$.

Las puérperas participantes consideran que el cuidarse hoy es la manera de asegurar su salud en el futuro, por lo que evitan durante esta etapa salir a la calle en las tardes, no alzar peso, ni realizar ejercicios domésticos, por el contrario, se considera propicio tomar bebidas calientes para garantizar el no enfermarse más adelante, ya que el puerperio es un periodo caliente y se debe evitar que entre frío al cuerpo concordante con los resultados obtenidos por Del toro y colaboradores ${ }^{1}$ estos cuidados culturales se mantienen pese a que por motivos de las restricciones sanitarias no existe un contacto directo de la puérpera con su grupo familiar de influencia como madre, abuela o suegra.
Los cuidados propiciados a los recién nacidos son importantes para evitar que se enfermen por lo que dentro de los principales cuidados mencionaron la lactancia materna para mantenerlos sanos, este hallazgo es congruente con la evidencia científica revisada ${ }^{1,23,24}$ sin embargo estudios realizados en pandemia ha encontrado que la falta de evidencia científica sobre el coronavirus ha afectado negativamente el establecimiento temprano de lactancia materna ${ }^{25}$

\section{Tema cultural: Perspectivas culturales del cuidado en el puerperio: cambio para dar respuesta a nuevas realidades}

Las acciones de cuidado hacen parte de lo que Leininger denomina sistemas populares 0 genéricos ${ }^{26}$ los cuales se debe negociar, reorientar y/o reestructurar para lograr un cuidado culturalmente congruente refiriéndose este, a los conocimientos, actos y decisiones de cuidado culturalmente usadas en formas sensibles $y$ reconocibles para ajustar apropiada y significativamente los valores, creencias y estilos de vida de los pacientes para su salud y bienestar, o para prevenir enfermedad, discapacidades 0 muerte $^{27}$ Suministrar este tipo de cuidado culturalmente coherente y seguro es el objetivo primordial de la Teoría del Cuidado Cultural, planteada por Leinninger. Acorde con lo anterior se propone lo siguiente:

\section{Creencias y prácticas que deberían preservarse}

Leinninger afirma que si la creencia o la práctica son beneficiosas o no representan daños pueden ser "preservados" ella define este tipo de prácticas como aquellos actos profesionales de asistencia, apoyo, facilitación, o habilitación o decisiones que ayuden a las culturas a retener, preservar, o mantener creencias y valores del cuidado o a enfrentar la discapacidad y la muerte.

El refuerzo positivo en lo relacionado a la lactancia materna y sus beneficios, prácticas que deben seguir con todas las medidas de bioseguridad como el lavado de manos para evitar la propagación de enfermedades incluida la covid-19 ${ }^{18}$, al igual que las 
prácticas de cuidado como el baño, la supervisión entre otros.

Es favorable el apoyo de la familia y en especial de las mujeres que ya tienen experiencia en el cuidado de los recién nacido, por lo que es necesario preservar esta práctica.

\section{Creencias y prácticas que deberían acomodarse o negociarse}

Si la creencia sobre la práctica puede llevar a algún riesgo, un acuerdo posiblemente apoyando la creencia debería ser "negociado", pero sugerido "o" acomodándose "una práctica más beneficiosa". Estas prácticas Leinninger las define como aquellas acciones creativas de asistencia, acomodación, facilitación o habilitación, del proveedor de cuidado, o decisiones que ayuden a las culturas a adaptar o negociar con otros buscando un cuidado congruente culturalmente, seguro y efectivo para su salud, bienestar, o para enfrentar la muerte o la enfermedad $^{28}$

Es preciso reforzar la autoestima de la puérpera a través del seguimiento de las buenas prácticas de cuidado y de la supervisión y apoyo de la familia, si no es posible de manera presencial por las actuales circunstancias, de forma telefónica.

\section{Creencias y prácticas que deberían reestructurarse o remodelarse}

Si la practicas son potencialmente nocivas, la enfermera debe adoptar una posición más firme en la explicación de los riesgos y la persona debe ayudarse en sustituir la práctica anterior por una más sana, reconociéndose siempre la autonomía y la decisión de la propia persona.

Leinninger las define como aquellas acciones profesionales y decisiones mutuas de asistencia, apoyo, facilitación o habilitación que puedan ayudar a las personas a reordenar, cambiar, modificar, o reestructurar sus estilos de vida en instituciones para mejorar los patrones de cuidado en salud, practicas o resultados ${ }^{27}$.

Es prioritario disminuir el estrés que puede aumentar las cifras de depresión posparto en la puérpera, además es necesario el direccionamiento desde el equipo de salud con programas de apoyo desde sus hogares.

\section{DECLARACIÓN SOBRE CONFLICTOS DE INTERÉS}

Los autores declaran la inexistencia de conflicto de intereses

\section{CONTRIBUCIÓN DE LOS AUTORES}

Primer autor: concepción del estudio, realización de entrevistas, análisis e interpretación de los datos y redacción inicial.

Segundo autor: búsqueda, revisión de la literatura, revisión crítica del manuscrito, aprobación final de la versión que será publicada.

Tercer autor: revisión crítica del manuscrito, discusión, redacción y aprobación finales de la versión que será publicada.

\section{REFERENCIAS BIBLIOGRÁFICAS}

1. De Dios-Pérez MI, Rojas-Carmona B, FernándezCarrasco FJ, Vázquez-Lara JM, Gómez-Salgado J, Rodríguez-Díaz L. Cuidados en el puerperio durante la crisis sanitaria por COVID-19. Arch Med (Manizales). 2021; 21(1): 300-304. Doi: https://doi.org/10.30554/archmed.21.1.3899.2021

2. Del Toro-Rubio M, Fernández-Aragón S, GonzálezRuiz G, Díaz-Pérez A, Aspectos culturales de la puérpera que influyen en el cuidado del primer hijo. Rev cubana Enfermer. 2016; 32(1): 37-48. Disponible en: http://scielo.sld.cu/scielo.php?script=sci_arttext\&p id=S0864-03192016000100006\&lng=es

3. Ruiz C. Cuidado Neonatal y Materno con Apoyo del grupo familiar. El Arte y La Ciencia del Cuidado. Bogotá: Grupo de Cuidado Facultad de Enfermería Universidad Nacional. Sede Bogotá; 2000

4. Marañón-Cardonne T, Mastrapa-Cantillo K, Poulut-Durades T, Vaillant-Lora L. COVID-19 y embarazo: Una aproximación en tiempos de pandemia. MEDISAN. 2020; 24(4): 707-727. Disponible en: 
http://scielo.sld.cu/scielo.php?script=sci_arttext\&p id=S1029-30192020000400707

5. Pérez-Banda A, Álvarez-Aguirre A, CasiqueCasique L, Díaz-Manchay R, Rodríguez-Cruz L. Cuidados culturales durante el puerperio mediato en las mujeres indígenas. 2019; 13(1):1313. Disponible en: http://scielo.isciii.es/scielo.php?script=sci_arttext\& pid=S1988-348X2019000100003\&lng=es

6. Bermejo-Sánchez FR, Peña-Ayudante WR, Espinoza-Portilla E. Depresión perinatal en tiempos del COVID-19: rol de las redes sociales en Internet. Acta Med Peru. 2020;37(1):88-93. Doi: https://doi.org/10.35663/ amp.2020.371.913

7. McFarland $M$, Wehbe-Alamah $H$. Leininger's transcultural nursing: Concepts, Theories research and practices. segunda edición.Usa: McGraw Hill.1995.

8. Salamanca-Castro A, Martín-Crespo C. El diseño en la investigación cualitativa. NURE investigación.2007 enero;26. Disponible en: https://dialnet.unirioja.es/servlet/articulo?codigo= 7787952

9. Spradley J. Participant Observation. Long Grove, Illinois:Waveland Press, Inc. 1983. Disponible en: https://books.google.com.co/books?hl=es\&lr=\&id= q7DICwAAQBAJ\&oi=fnd\&pg=PR3\&dq=Spradley, + Ja mes+P.+(1980).+Participant+observation.+New+Yo rk:+Holt,+Rinehart+and+Winston.\&ots $=\mathrm{H}-$

dmMUPQ39\&sig=Rsjhf72qMciYaf4mRIKjplp5U0k\#v $=$ onepage $\& q \& f=$ false

10. Leininger $\mathrm{M}, \mathrm{Mc}$ Farland $\mathrm{M}$. Culture care diversity and universality. Second edition. Sudbury, Massachusetts: Jones and Bartlett Publishers; 2006. Disponible

en: https://books.google.com.co/books?hl=es\&lr=\&id= $\mathrm{NmY43MysbxIC \& oi=fnd \& pg=PR5 \& dq=Leininger,+M}$ adeleine $+M+y+M a c f a r l a n d+R .+M a r i l y n+U n i v e r s a l i t$ $\mathrm{y}$ +and+diversity+of+cultural+care.\&ots=Jmbok84bf Y\&sig=OPP_ayZgp1e3ONI_8rRHhvfZNtk\#v=onepag e\&q=Leininger\%2C\%20Madeleine\%20M\%20y\%20 Macfarland\%20R.\%20Marilyn\%20Universality\%20a nd\%20diversity\%20of\%20cultural\%20care.\&f=false
11. Fornons-Fontdevila, D. Madeleine Leininger: claroscuro trascultural. Index Enferm. 2010; 2-3(19): 172-176. Disponible

en: http://scielo.isciii.es/scielo.php?script=sci_arttext\& pid=S1132-12962010000200022\&lng=es

12. Hernández-Sampieri R, Fernández-Collado C, Baptista-Lucio P. Metodología de la Investigación. Quinta edición. México D.F.: McGraw-Hill;2010. Disponible en https://www.icmujeres.gob.mx/wpcontent/uploads/2020/05/Sampieri.Met.Inv.pdf

13. McCurdy D, Spradley J, Shandy D.The cultural experience: Ethnography in Complex Society. Second edition; 2005. E.U.: Waveland Press,Inc; 2005.Disponible en: https://books.google.com.co/books?id=XWobAAA AQBAJ\&Ipg=PR1\&ots=dQzqjdhKB_\&dq=The\%20cult ural\%20experience\%3A\%20Ethnography\%20in\%20 Complex\%20Society\&lr\&hl=es\&pg=PR1\#v=onepage $\& q=$ The\%20cultural\%20experience:\%20Ethnograph y\%20in\%20Complex\%20Society\&f=false

14. Spradley J. The Ethnographic Interview. New York : Holt, Rinehart and Winston; 1979. Disponible en: https://www.worldcat.org/title/ethnographicinterview/oclc/4493439\#borrow

15. Salgado-Lévano A, Investigación cualitativa: Diseños, Evaluación de Rigor Metodológico y Retos. liber. 2007; 13 (13): 74-76. Disponible en: http://www.scielo.org.pe/scielo.php?pid=S172948272007000100009\&script=sci_arttext\&tIng=en

16. Colombia. Ministerio de Salud. Resolución № 008430, Por la cual se establecen las normas científicas, técnicas y administrativas para la investigación en salud. República de Colombia;1993. Diario oficial. Disponible en: https://www.minsalud.gov.co/Normatividad_Nuev o/RESOLUCION\%208430\%20DE\%201993.pdf

17. Muñoz-De Rodríguez L, Vásquez ML. Mirando el cuidado cultural desde la óptica de Leininger. Corporación Editorial Médica del Valle. Colombia Médica. 2007;38(4):98-104. Disponible en: https://core.ac.uk/download/pdf/11863863.pdf 
18. Mascarenhas VHA, Caroci-Becker A, Venâncio KCMP, Baraldi NG, Durkin AC, Riesco MLG. Care recommendations for parturient and postpartum women and newborns during the COVID-19 pandemic: a scoping review. Rev. Latino-Am. Enfermagem. 2020;28:

https://doi.org/10.1590/1518-8345.4596.3359

19. Franco-Ramírez J, Cabrera-Pivaral C, ZárateGuerrero G, Franco-Chávez S, CovarrubiasBermúdez M, Zavala-González M. Representaciones sociales de adolescentes mexicanas embarazadas sobre el puerperio, la lactancia y los cuidados del recién nacido. Bol. Med. Hosp. Infant. Mex. 2018 Junio;75

(3):153-159. https://doi.org/10.24875/bmhim.m18000014.

20. Leininger M. Ethnography and ethnonursing: Models and modes of qualitative data analysis. Orlando, Fl: Grune \& Stratton;1985.

21. Madge $\mathrm{C}, \mathrm{O}^{\prime}$ Connor $\mathrm{H}$. Parenting gone wired: empowerment of new mothers on the internet? Soc Cult Geogr. 2006;7(2):199-220. Doi: 10.1080/14649360600600528. Disponible en: https://www.tandfonline.com/doi/full/10.1080/14 649360600600528?casa_token=mWaJt4A58XsAAA AA\%3AHZvAN945SpJXd5TADW1hXMBMoQMfVsTX W6DzRbTcK60Qc4BpSnHiOc87CW7xhRNYMsROOrNF6cT

22. Moon RY, Mathews A, Oden R, Carlin R. Mothers' Perceptions of the Internet and social media as Sources of Parenting and Health Information: Qualitative Study. J Med Internet Res. 2019;21(7):e14289. Doi: 10.2196/14289. Disponible en: https://www.jmir.org/2019/7/e14289/

23. Brahm P, Valdés $V$. Beneficios de la lactancia materna y riesgos de no amamantar. Rev. chil. pediatr. 2017;88(1):07-14. http://dx.doi.org/10.4067/S0370-

41062017000100001

24. Aguilar-Cordero M, Baena-García L, SánchezLópez A, Guisado-Barrilao R, Hermoso-Rodríguez E, Mur-Villar N. Beneficios inmunológicos de la leche humana para la madre y el niño: revisión sistemática. Nutr. Hosp. 2016.Abril; 33(2): 482-493. http://dx.doi.org/10.20960/nh.526.
25. Vila-Candel R, Mena-Tudela D, Gómez-Seguí A, et al. Manejo del parto, el puerperio y la lactancia en mujeres positivas para SARS-CoV-2. Estudio multicéntrico en la Comunidad Valenciana. Enferm Clin. 2021 May-June; 31(3): 184-188. doi: 10.1016/j.enfcli.2021.01.006

26. Fornons- Fontdevila D. Madeleine Leininger: Claroscuro Transcultural. Index Enferm. 2010; 2-3 (19):172-176. Disponible en: http://scielo.isciii.es/scielo.php?pid=S113212962010000200022\&script=sci_arttext

27. Escobar-Castellanos B, Paravic-Klijn T. La transculturalidad, elemento esencial para mejorar la atención en salud y de enfermería. Revenf. 2017; 32. Disponible en: https://www.scielo.sa.cr/pdf/enfermeria/n33/1409 -4568-enfermeria-33-00073.pdf

28. Leininger M. Ethnography and ethnonursing: Models and modes of qualitative data analysis. Orlando, Fl: Grune \& Stratton;1985. 